\title{
Distributed Generation Integration in the Electric Grid: Energy Storage System for Frequency Control
}

\author{
Maurizio Delfanti, Davide Falabretti, Marco Merlo, and Gabriele Monfredini \\ Department of Energy, Politecnico di Milano, 20156 Milan, Italy \\ Correspondence should be addressed to Marco Merlo; marco.merlo@polimi.it
}

Received 31 January 2014; Revised 5 May 2014; Accepted 13 May 2014; Published 11 June 2014

Academic Editor: Ned Djilali

Copyright ( 2014 Maurizio Delfanti et al. This is an open access article distributed under the Creative Commons Attribution License, which permits unrestricted use, distribution, and reproduction in any medium, provided the original work is properly cited.

During the last few years generation from renewable energy sources (RESs) has grown considerably in European electrical networks. Transmission system operators are greatly concerned about the impact of RESs on the operational security and efficiency of their networks and more in general of the ENTSO-E interconnected system. Grid codes are to be revised in order to harmonise the rules regarding the connection of RES power plants. A main issue concerns frequency control: frequency is greatly affected by RESs intermittency and its deviations must be limited as much as possible in order to guarantee a suitable level of power quality. To improve frequency stability, in the future, Grid codes could extend frequency control requirements also to RES units, whereas today they are applied only to conventional power plants. Energy storage systems can be a possible solution to increase the flexibility and performance of RES power plants: they allow generators to modulate their power injections without wasting renewable energy. In this paper, the authors studied the suitability of extending frequency control to RES units integrating them with energy storage systems. In particular, the paper focuses on the impact of frequency control on the storage lifetime by analysing the power charge/discharge in response to real frequency oscillations.

\section{Introduction}

Renewable Energy Sources (RESs) are essential in the perspective of a green, fully sustainable, and energetic approach. However, a high RES penetration introduces technical issues and challenges many aspects of the operation of power systems (e.g., power quality, system reliability and safety, and control and protection strategies) [1]. Distributed generation (DG) is not dispatchable like conventional generation and does not provide any ancillary service to the system; therefore, a large amount of DG stresses the power system operation, bringing it closer to the stability margin. In particular, the higher the percentage of DG is, the lower the reserve for the primary frequency regulation becomes. This fact is highlighted by Figure 1, which shows the primary frequency regulation reserve in the Italian electrical system: in the last four years the increase of RES generation has caused the reserve to decrease up to $20-25 \%$ (e.g., for the year 2012 a primary reserve equal to $500 \mathrm{MW}$ corresponds to a duration time, the grey dashed line in Figure 1, which for the year 2008 had been characterised by a reserve power of $600 \mathrm{MW}$ ).
Storage apparatuses could be a challenging option in order to cope with the problem; that is, they could be adopted to mitigate the frequency fluctuations in order to guarantee adequate power quality and security of supply levels [2].

Concerning ancillary services support, RES power plants connected to the medium voltage (MV) and low voltage (LV) distribution system do not allow an easy and feasible remote controllability; the installation of a proper communication system may require long-term investments and large amounts of capital. Nevertheless, focusing on the primary frequency regulation, one way to address this issue over a short time period is to adopt a local frequency control that exploits the frequency measurements at the DG's point of common coupling (PCC), as a feedback of the status of the system; reacting to this signal DG could make a power margin available to restore the power imbalance. It means an exploitation of local measurements to solve a global issue.

Actually, the problem is well known and deeply investigated in the literature, from the regulatory, technical, and economic point of view: [3] reports an international overview of the already in place practices concerning ancillary services, 


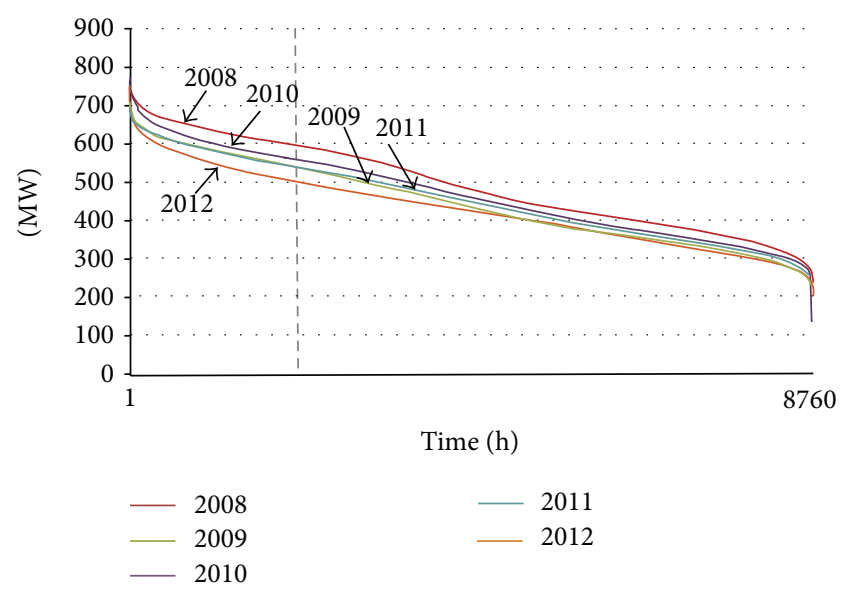

FIGURE 1: Frequency primary reserve duration curves in the Italian system: 2008-2012 figures [8].

and $[4,5]$ evaluate the possible integration of these services in the liberalised market structures. References [5-7] deeply investigate the market and policy barriers/opportunities in order to manage effectively the ancillary services, exploiting also the possible contribution of passive users (i.e., demand side management functions).

The goal of this paper is to focus on the DG role with respect to ancillary services management, with particular reference to the primary frequency control.

Figure 2 depicts the distribution of DG power plants connected to the Italian system, according to RES and size; there is clear evidence that the exploitation of the Photovoltaic (PV) renewable source is usually performed by small-size power plants (lower than $50 \mathrm{~kW}$ ) connected to the MV and LV distribution system. In 2012, the total amount of PV generation in the Italian system was about $16 \mathrm{GW}$ [9].

In order to achieve suitable network reliability, in the future DG will have to provide the primary frequency regulation service. If a retrofit of all PV power plants already in place in Italy were put in place $(16 \mathrm{GW})$, by making available, for example, $3 \%$ of the rated power for the regulation, it would be possible to recover $480 \mathrm{MW}$ of primary frequency reserve. This way a proper regulation capability would be reestablished and the system stability during frequency oscillations would be considerably improved.

Generally speaking, a key factor involves the metrics designed in order to define planning and operational requirements in an electric power system characterised by high penetration of RESs [10].

In this scenario, an effective solution could be to integrate energy storage systems (ESSs) into DG power plants: ESSs could improve the frequency regulation ensuring the energy needed by the frequency control. Furthermore, ESSs cope better with frequency control because they exhibit a rapid active power response; so they are ideal to compensate the generation rate constraints of slower generators and the fluctuations produced by RESs.

Electric energy can be stored electromagnetically, electrochemically, kinetically, thermally, or as potential energy. Two factors characterise the application of an energy storage

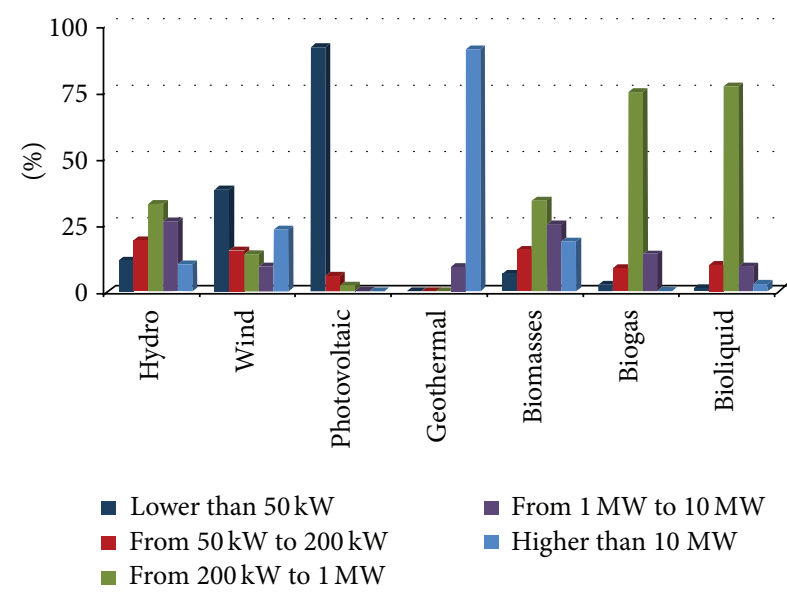

FIGURE 2: Distribution [\%] of the power plants connected to the Italian system according to RES and size [9].

technology: one is the amount of energy that can be stored in the device and the other is the rate at which energy can be transferred into or out of the storage device. These factors are mainly determined by the characteristics of the ESS itself $[1,11]$.

The primary frequency control service provided by an integrated system of DG and ESS requires "power performances" to make energy available over a short time period (i.e., a high ratio between the power injected and the energy stored).

The basic issues regarding storage technologies consist of the cost of these technologies, and their operational and maintenance requirements [12]. Since a large-scale ESS is rather expensive, defining requirements for the frequency control service can become a critical issue; it is also evident that the application should be tailored to minimise the ESS size needed.

Another important issue regarding the ESS is the state of charge (SoC) control. It can be shown that the ESS can easily be depleted or overcharged if no additional control actions are performed [13].

The converter adopted for the connection of the ESS to the network plays a key role in the definition of the power set-point needed for the frequency regulation. Different bidirectional converter structures are reported in the literature, while the control methods make the difference in the ESS's dynamic performances [14]. New DG units, mainly PV and wind generators, are usually coupled with the ESS by means of a DC-coupled system. In this structure, the power plant and the storage are connected to a common DC bus (called DClink) by proper DC/DC converters; the system is interfaced to the AC network through a common DC/AC inverter. This is the simplest and cheapest solution for the integration of the ESS, because it requires the minimum number of converters and therefore the minimisation of the power losses. On the contrary, in an AC-coupled system, the generator and the ESS are connected at the $\mathrm{AC}$ level, requiring a $\mathrm{DC} / \mathrm{AC}$ inverter and a DC/DC converter for each component; therefore, this scheme implies higher investments and operational costs. 
In the Italian framework, technical regulations already proposed a contribution of DG power plants to ancillary services (CEI 0-16 [15]). In particular, some issues, such as the low voltage fault ride through and the reactive power modulation, are mandatory; others, such as the contribution to the frequency regulation, are at present being defined (several consultation processes are ongoing). In order to develop an effective technical regulation, it is increasingly important to develop realistic analyses concerning the ancillary services requirement for the electric grid and the impact of the ancillary services support on the DG. This paper focuses on the latter point: in particular the integration of an ESS in a PV power plant and the impact of the frequency regulation on the storage device are evaluated. The final goal is to provide realistic data useful for the ESS design. The paper is structured as follows: Section 2 investigates the primary frequency regulation concept; Section 3 describes the proposed control scheme and the ESS sizing; the study case for the numerical analysis and the test results are described in Section 4; and, finally, proper conclusions are reported in Section 5.

\section{Primary Frequency Regulation Proposed for DGs Coupled with ESS}

Power system generation portfolios are changing rapidly, and the inertial and dynamic characteristics of many new sources of electric generation differ from those of the past. The lack of predictable and controllable energy sources, together with the different operating characteristic of DG power plants with respect to their inertial response, poses challenges to transmission system operators (TSOs) trying to control system frequency [16].

In order to address the need to include DG power plants, mainly connected to distribution networks, in the primary frequency control system, the ENTSO-E grid code [17] suggests the primary frequency regulation strategy reported in Figure 3: the real power is modulated as function of the frequency deviation from the nominal value. The curve is characterised by the following parameters.

(i) Frequency response deadband: the real power is not modulated in the case of small frequency deviations from the nominal value.

(ii) Droop control: during underfrequency deviations the generator has to provide a positive real power output change according to a droop strategy and, on the contrary, during overfrequencies a negative power change has to be provided.

(iii) Maximum power capability: the maximum power that the generator is capable of providing.

The proposed scheme represents a local strategy for primary frequency regulation, which is possible only by measuring the frequency oscillations at the PCC. It is important to point out that, in the perspective of each single generation unit, the primary frequency service does not require a large amount of energy/power; the grid code prescriptions typically range from $1.5 \%$ to $3 \%$ of the generator rated power,

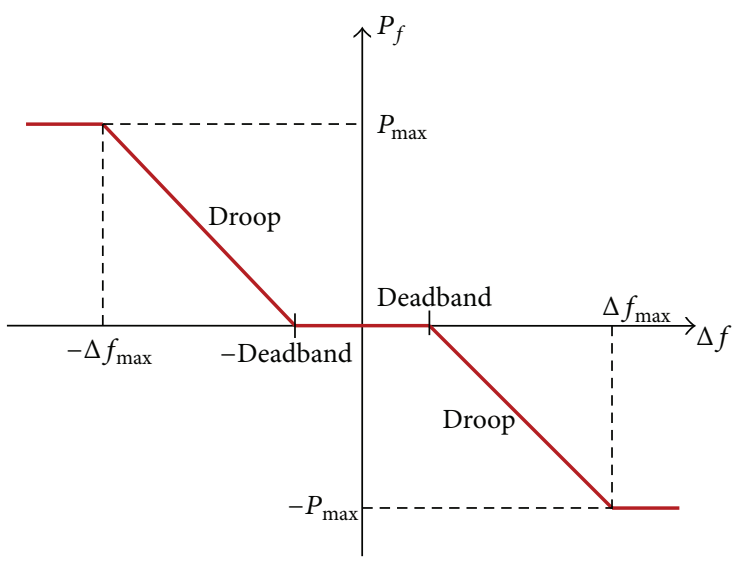

FIGURE 3: Local strategy for the primary frequency regulation.

over a time ranging from 15 minutes to 1 hour. The real power frequency response is activated as fast as technically feasible, with an initial delay that is as short as possible (this delay will be reasonably justified to the TSO, if greater than 2 seconds). The maximum time of full activation has to be 30 seconds.

It is worth noting that, from the main grid perspective, the collection of the regulating contribution from all the generating units provides a significant amount of power, useful to guarantee the system stability and security.

In this work a mathematical model of an ESS integrated in a PV plant is developed and connected to a simplified test network. A combination of static devices and ESSs has been proposed; proper control strategies are necessary in order to achieve full benefits from these technologies. A real frequency oscillation is superimposed on the model. The output of the simulation represents the behaviour of the single storage module in response to the frequency regulation.

The approach proposed is based on the assumption that, locally (i.e., for each DG unit), the frequency control support (i.e., rated at $1.5 \%-3 \%$ of the generation unit nominal power) could be provided by exploiting the ESS; in particular, technically speaking, the same prescriptions already in place for conventional power units have been assumed. In such a way, the main grid behaviour is not supposed to change; that is, an increase in DG penetration, replacing the conventional power plants, could be effective thanks to maintaining the same ancillary service support we had in the past.

\section{Storage Integration Control Scheme}

In this study RMS simulations are carried out considering the dynamic model, built in the DIgSILENT PowerFactory software environment, reported in Figure 4. The model is a DC-coupled system and operates in a grid-connected mode.

The PV and the ESS are connected to a simplified MV distribution network through an MV/LV transformer (INVERTER-Trafo) and a DC/AC converter (INVERTER) in a grid-following mode operation; therefore, the model requires a main grid as frequency reference for the control of its outputs.

In the configuration, the PV system, the ESS, and the AC network are connected to the DC-link by means of 


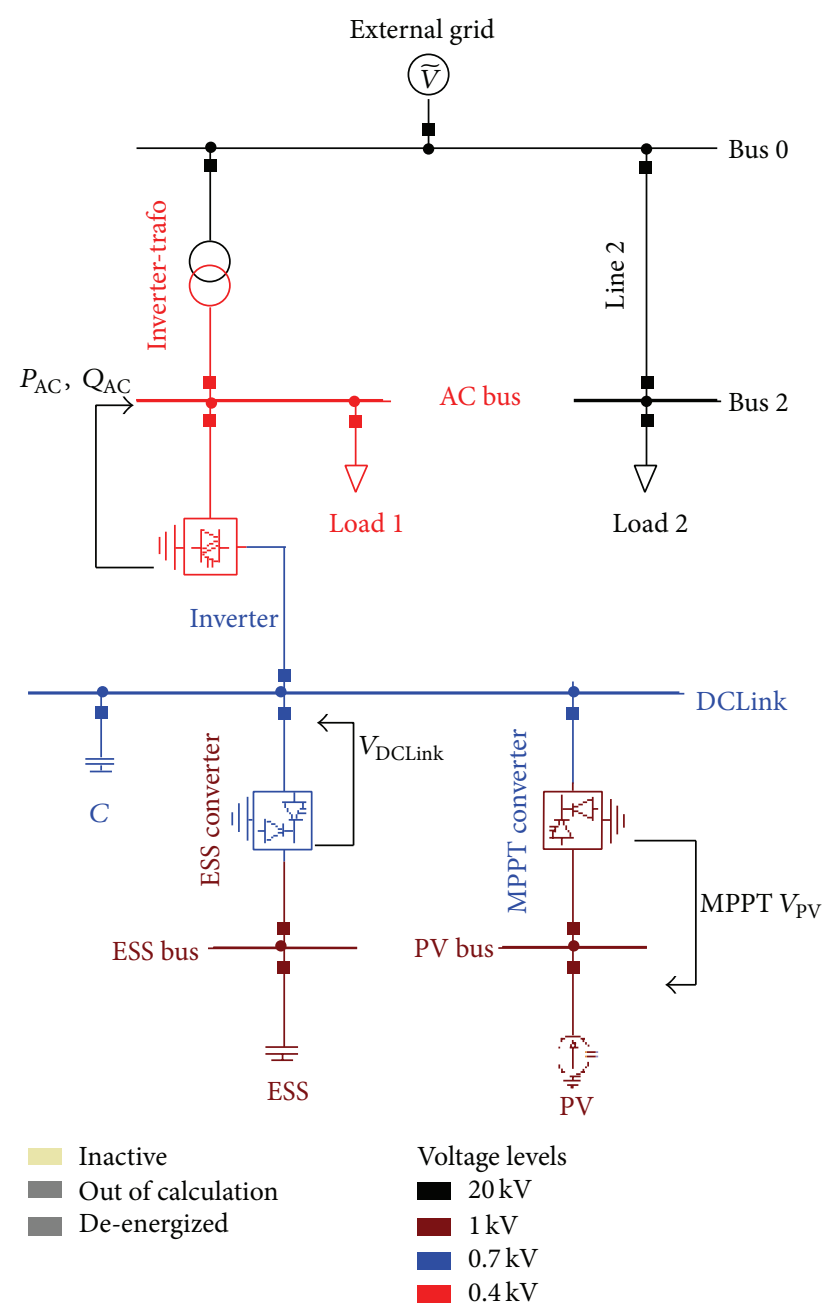

FIGURE 4: Network scheme of the PV system integrated with an ESS in a DC-coupled system.

appropriate converters, in order to match the PV and ESS voltages and to control the AC side independently. The PV system is connected to the common DC bus through a DC-DC converter (maximum power point tracking (MPPT) converter) to ensure the optimal power exchange; whereas the ESS is connected using a bidirectional DC-DC converter (ESS converter) to guarantee the charge and discharge of the storage device.

In the following the functions of each component presented in the scheme are listed.

(i) The PV system injects a constant power equal to its own optimal power $\left(P_{\mathrm{MPPT}}\right)$.

(ii) The MPPT converter controls the voltage of the PV system $V_{\mathrm{PV}}$ at its own MPPT.

(iii) The INVERTER controls the real power $P_{\mathrm{AC}}$ and reactive power $Q_{\mathrm{AC}}$ injected at the $\mathrm{AC}$ side.

(iv) The ESS converter controls the voltage at the DC-link $\left(V_{\text {DCLink }}\right)$ in order to set the power balance at the DClink (1). (v) The ESS injects/absorbs the power required to match the power balance at the DC-link according to the ESS converter control.

The power balance equation at the DC-link is

$$
P_{\mathrm{PV}}-P_{\mathrm{ESS}}-P_{\mathrm{AC}}=C \cdot V_{\mathrm{DCLink}} \cdot \frac{d V_{\mathrm{DCLink}}}{d t},
$$

where $P_{\mathrm{PV}}$ is the power produced by the PV system (controlled to a set-point equal to the MPPT power $P_{\mathrm{MPPT}}$ ); $P_{\mathrm{ESS}}$ is the power absorbed by the ESS; $P_{\mathrm{AC}}$ is the power injected to the AC network by the inverter; $C$ is the capacitance of the DC-Link; and $V_{\text {DCLink }}$ is the voltage measured at the DCLink.

The $P_{\mathrm{PV}}$ power is assumed constant and represents an external and noncontrollable value of the power balance equation. The injected power $P_{\mathrm{AC}}$ is determined by the $\mathrm{PV}$ power $P_{\mathrm{PV}}$ and the power required for the frequency control. Since $P_{\mathrm{PV}}$ and $P_{\mathrm{AC}}$ are external inputs in (1), the power provided by the ESS $P_{\text {ESS }}$ is exploited to compensate the power mismatch between the $\mathrm{PV}$ production and the injection to the AC side.

On the basis of the aforementioned functions, a numerical model of the proposed system has been developed and the control laws of each converter are defined. For this purpose, the following assumptions are considered:

(i) all converters are ideal (losses are not modelled);

(ii) static switches commutation is not modelled and only a model at fundamental frequency is taken into account (assumption in accordance with the RMS dynamic);

(iii) the DC-link capacitor is modelled as an ideal capacitance (the losses and the auto-discharge phenomena are not considered);

(iv) the ESS is modelled as a passive first order dynamic model;

(v) the PV power plant injects a constant power.

In the following sections a detailed description of each component is given.

3.1. PV System. The PV system is modelled as a constant DC current source with a parallel internal conductance. During the simulation the current source injects a constant current and the $V_{\mathrm{PV}}$ voltage is controlled by the MPPT converter to meet the optimal power injections of the PV.

3.2. MPPT Converter. The MPPT converter is a DC-DC converter that regulates the $V_{\mathrm{PV}}$ voltage to its MPPT set-point $V_{\text {MPPT }}$. In this way it is possible to extract the maximum power available from the PV power plant $\left(P_{\mathrm{MPPT}}\right)$ and at the same time to decouple the voltage $V_{\mathrm{PV}}$ and the DC-link voltage $V_{\text {DC-Link }}$. The control is achieved by means of a PI regulator. The output of the regulator is the $\alpha$ ratio, used as control variable for the MPPT converter:

$$
\alpha=\frac{V_{\mathrm{DCLink}}}{V_{\mathrm{PV}}} .
$$


3.3. INVERTER. The system is connected to the AC network through an AC-DC inverter (INVERTER). This device provides an interface to the ESS and the PV system for real and reactive power exchanges with the power system. The INVERTER is an ideal, lossless, PWM converter modelled as a DC-voltage controlled AC-voltage source:

$$
V_{\mathrm{AC}}=\left(P_{\mathrm{mr}}+j P_{\mathrm{mi}}\right) \cdot V_{\text {DC-Link }},
$$

where $V_{\mathrm{AC}}$ is the AC voltage phasor, $V_{\mathrm{DC}-\text { Link }}$ is the voltage at the common DC bus, and $P_{\mathrm{mr}}$ and $P_{\mathrm{mi}}$ are the pulse modulation factors on the real and imaginary axes.

If a $d$ - $q$ reference-system fixed to the $\mathrm{AC}$ voltage phasor is introduced, a decoupled $P-Q$ control can be obtained (as detailed in [18]). This way the real power $P_{\mathrm{AC}}$ is determined only by the $d$ current component $I_{\mathrm{AC} d}$, whereas the reactive power $Q_{A C}$ is determined only by the $q$ current component $I_{\mathrm{AC} q} ; P_{\mathrm{AC}}$ and $Q_{\mathrm{AC}}$ can be controlled independently. The control scheme adopted for the inverter is shown in Figure 5.

Two control loops are employed by the INVERTER in order to control the real and reactive power injected at the AC side:

(i) the outer loop controls $P_{\mathrm{AC}}$ and $Q_{\mathrm{AC}}$ by defining the current set-points $I_{\mathrm{AC} d \text {-ref }}$, and $I_{\mathrm{AC} q \text {-ref; }}$

(ii) the inner loop controls $I_{\mathrm{AC} d}$ and $I_{\mathrm{AC} q}$ by defining the pulse modulation factors $P_{\mathrm{m} d}$ and $P_{\mathrm{m} q}$ used by the PWM technique of the inverter.

The outer control loop employs slow bandwidth PI regulators, whereas the PI regulators of the inner loop are usually designed to achieve faster bandwidths [19]. Proper $d$ $q$ transformations ( $r-i$ to $d-q$ ) and antitransformations ( $d-q$ to $r-i)$ are adopted to compute $I_{\mathrm{AC} d}, I_{\mathrm{AC} q}, P_{\mathrm{mr}}$, and $P_{\mathrm{mi}}$; a phase lock loop (PLL) is exploited to measure the angle $\theta$ of the AC voltage that is necessary for the axes transformations.

The overall control has three operational objectives:

(i) to perform the normal inverter operation by injecting the power produced by the PV system $\left(P_{\mathrm{MPPT}}\right)$;

(ii) to provide the primary frequency control using a droop control;

(iii) to maintain the state of charge (SoC) of the ESS to a predefined value (by means of the $\mathrm{SoC}$ restoration function).

The inverter controls the real power $P_{\mathrm{AC}}$ with the following set-point:

$$
P_{\mathrm{ACref}}=P_{\mathrm{MPPT}}+P_{f} \cdot K+\mathrm{DP}_{\mathrm{refESS}} \cdot(1-K) .
$$

The reactive power is forced to zero (the system injects power at power factor equal to one). The set-point $P_{\mathrm{ACref}}$ of (4) is represented in the block scheme of Figure 6.

(i) $P_{\mathrm{MPPT}}$ is the MPPT power of the PV system; this is the power injected by the inverter during normal conditions (i.e., with no frequency oscillations or critical conditions for the ESS's SoC); (ii) $P_{f}$ is the power change due to the frequency deviation $\Delta f$ with respect to the nominal value $f_{n}(\Delta f=$ $f-f n)$ computed according to the local strategy of Figure 3, where:

(a) $P_{\max }$ : maximum power capability available for the control,

(b) deadband: frequency range of no power modulations,

(c) droop: slope of the section of the curve with power modulation $(\Delta f / \Delta P$ ratio $)$.

(iii) $\mathrm{DP}_{\text {refEss }}$ is a set-point component which leads to the restoration of the SoC of the ESS at the nominal value according to the $\mathrm{SoC}$ restoration function, as explained in the next section.

(iv) $K$ is the flag for the selection among the $P_{f}$ and the $\mathrm{DP}_{\text {refESS }}$ set-points; in particular it disables the frequency control enabling the SoC restoration function or vice versa according to the $\mathrm{SoC}$ and frequency oscillation entities.

3.4. SoC Restoration Function. In order to operate the system, the charging level of the ESS has to be regulated not to exceed its operational range, that is, not to hit the maximum/minimum allowed SoC. Reference [20] demonstrates the impact of the SoC restoration function on the ESS design and operation, and in particular it shows that the ESS capacity can be reduced by a high recharge percentage available for this function. In the work proposed, an SoC restoration function has been introduced in the control to keep the SoC of the storage within its proper range while the storage provides the frequency regulation service. The SoC restoration function is activated during small frequency oscillation periods without a degradation of the system frequency quality. To this purpose, the additional charging reference $\mathrm{DP}_{\text {refESS }}$ to the $\mathrm{AC}$ power set-point (as depicted in the block diagram of Figure 6) is introduced. The implemented $\mathrm{SoC}$ restoration function has the following characteristics:

(i) it reestablishes the target $\mathrm{SoC}(50 \%)$ when the frequency is inside a noncritical window (i.e., the deadband);

(ii) it uses a small recharge power (a few percentage of the rated power);

(iii) if the storage reaches the SoC limits, the function is activated no matter what the frequency value is.

The target level of the SoC is assumed in a range between the maximum SoC limit (80\%) and the minimum SoC limit $(20 \%)$ in order to keep a proper charging reserve for facing both overfrequency (ESS absorption) and underfrequency events (ESS injection).

The $K$ flag of (4) allows the activation of the SoC restoration set-point as an alternative to the frequency control 


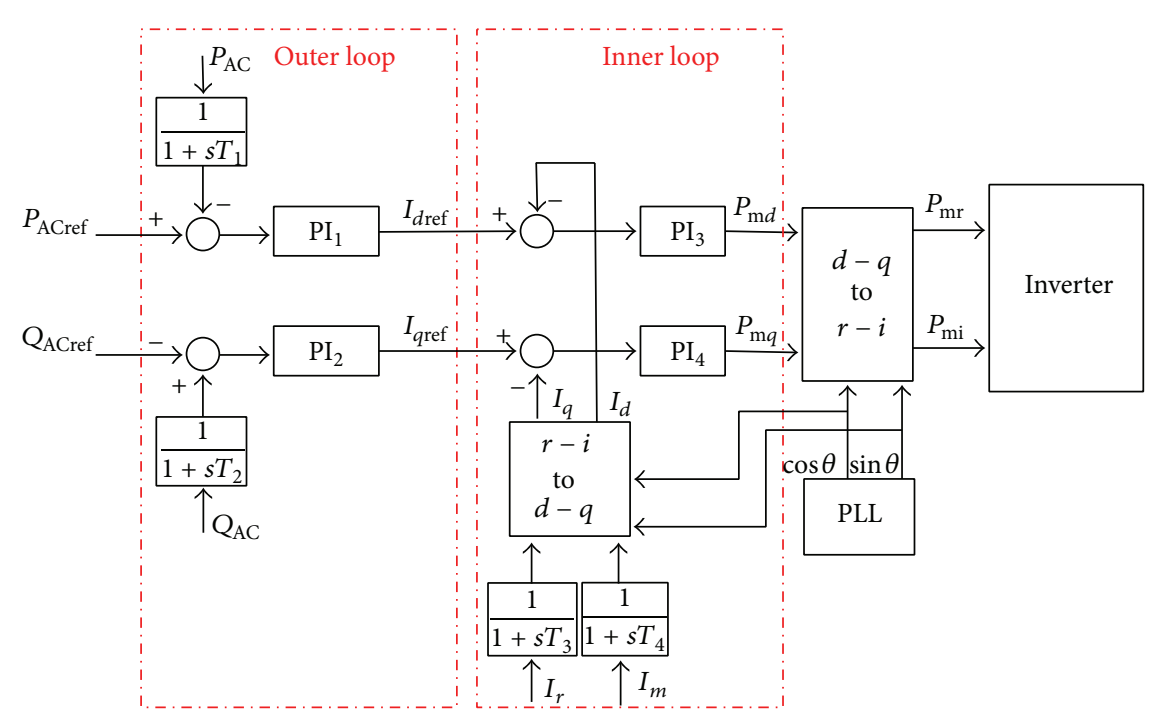

Figure 5: Control frame of the INVERTER.

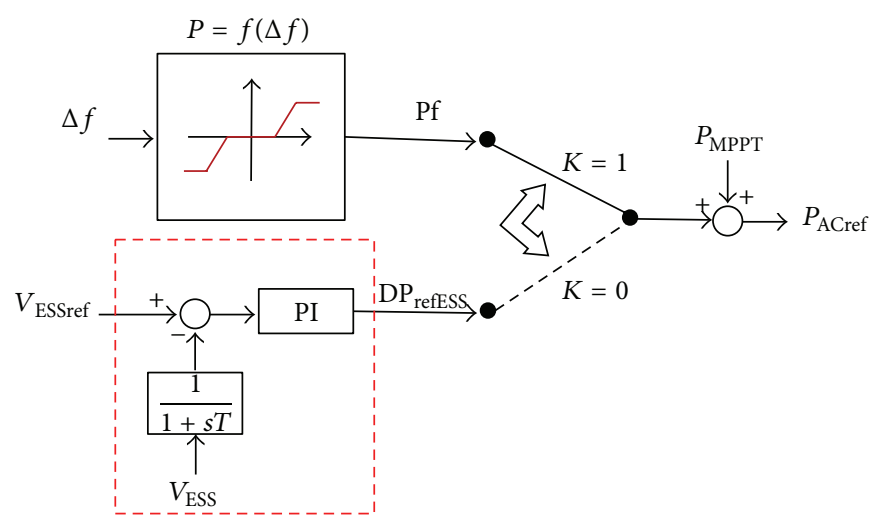

FIGURE 6: Control frame for the definition of the AC real power set-point $P_{\text {ACref }}$. The red box identifies the SoC restoration function.

set-point $P_{f}$. In order to define it, the ESS saturation flag $K_{\text {ESSSat }}$ is introduced as follows:

$$
K_{\text {ESSSat }}= \begin{cases}V_{\mathrm{ESS} \min } \leq V_{\mathrm{ESS}} \leq V_{\mathrm{ESS} \max } & \text { for } 10 \mathrm{~s}, \\ V_{\mathrm{ESS}}>V_{\mathrm{ESS} \max } \text { or } V_{\mathrm{ESS}}<V_{\mathrm{ESS} \min } & \text { for } 10 \mathrm{~s} .\end{cases}
$$

The ESS is modelled as an $R$-C series and its voltage $V_{\text {ESS }}$ is the variable directly linked with the SoC: when the maximum SoC limit is reached the maximum voltage limit $V_{\text {ESS max }}$ is also reached, and when the minimum SoC limit is reached the minimum voltage limit $V_{\text {ESS min }}$ is also reached. Therefore, $K_{\text {ESSSat }}$ is enabled when the ESS is completely charged (maximum SoC) or discharged (minimum SoC) and it cannot make available energy for the primary frequency control. A temporal hysteresis of $10 \mathrm{~s}$ is introduced for the activation and deactivation of this functionality in order to prevent instability. Based on (5), the $K$ flag is defined as follows:

$$
K= \begin{cases}1 & \text { for } K_{\text {ESSSat }}=1,|\Delta f|>\text { deadband, } \\ 0 & \text { for } K_{\text {ESSSat }}=0 \text { or }|\Delta f|<\text { deadband. }\end{cases}
$$

Equations (5) and (6) allow the attainment of the aforementioned SoC restoration function. As shown in the block diagram of Figure 6 , the set-point $\mathrm{DP}_{\text {refESs }}$ is generated by a $\mathrm{PI}$ regulator; this component is limited to the value $\mathrm{DP}_{\max }$, which is much lower than that of $P_{\max }$, and it has no significant effect on the frequency oscillations.

Finally, in order to prevent important contingencies, that is, events driving a significant frequency deviation, from overcharging the ESS thereby causing fast SoC variations, upper and lower bounds to the power output $\left(P_{\max }\right.$ and $P_{\min }$ in Figure 3) have been introduced; vice versa, the SoC restoration function does not directly manage these events.

This issue is not considered critical, in fact, severe contingencies are events with a low probability and, in modern power systems, are managed collecting the regulating contributions from all the resources available or by specific functions (e.g., emergency condition load shedding). Actually, the proposed DG and ESS coupling would respond like a conventional power plant, supporting the system up to its capability limits, $P_{\max }$ and $P_{\min }$. 
3.5. ESS Converter. The ESS Converter is a bidirectional DCDC buck-boost converter, which controls the $V_{\text {DC-Link }}$ voltage by charging and discharging the ESS module. The converter is controlled varying the duty ratio $\alpha$ by means of a PI regulator. The primary objective of the control of the ESS Converter is to maintain the power balance at the DC-Link (1) by means of the energy stored in the ESS.

The DC-link voltage $V_{\text {DCLink }}$ is compared with the reference $V_{\text {DCLink-Ref }}$ and the error is taken into a PI regulator that outputs the reference current $I_{\mathrm{DC}-\mathrm{Link}}$, which is absorbed at the DC-link by the converter (Figure 6) [19]. During the boost mode the ESS provides energy to the DC-link, whereas in the buck mode the ESS is charged in order to compensate the power mismatch between the real power output of the INVERTER and the PV system.

The model is completed with the power balance equation

$$
\begin{gathered}
P_{\mathrm{ESS}}=V_{\mathrm{ESS}} \cdot I_{\mathrm{ESS}}=V_{\mathrm{DC}-\text { Link }} \cdot I_{\mathrm{DC}-\text { Link }}, \\
\alpha=\frac{V_{\mathrm{DC}-\text { Link }}}{V_{\mathrm{ESS}}}=\frac{I_{\mathrm{ESS}}}{I_{\mathrm{DC}-\text { Link }}},
\end{gathered}
$$

where $V_{\text {ESS }}$ and $I_{\text {ESS }}$ are the voltage and current measured at the ESS bus; $V_{\mathrm{DC} \text {-Link }}$ and $I_{\mathrm{DC} \text {-Link }}$ are the voltage and current measured at the DC-Link; and $\alpha$ is the duty ratio of the ESS converter.

During underfrequencies, the INVERTER injects in the network an amount of power equal to $P_{\mathrm{MPPT}}+P_{f}$ with $P_{f}$ positive (obtained by the primary frequency control curve); since $P_{\mathrm{MPPT}}$ is supplied by the PV system, $P_{f}$ is delivered by the ESS. On the contrary, during overfrequencies $P_{f}$ is negative and the ESS is charged.

3.6. ESS Model. The ESS is modelled through an ideal capacitance and an equivalent series resistance; the dynamic model is:

$$
\begin{gathered}
V_{\mathrm{ESS}}=V_{\mathrm{C}}+R \cdot I_{\mathrm{ESS}}=V_{\mathrm{C}}+R \cdot \frac{P_{\mathrm{ESS}}}{V_{\mathrm{ESS}}}, \\
\frac{d V_{C}}{d t}=\frac{1}{C_{\mathrm{ESS}}} \cdot I_{\mathrm{ESS}}=\frac{1}{C_{\mathrm{ESS}}} \cdot \frac{P_{\mathrm{ESS}}}{V_{\mathrm{ESS}}},
\end{gathered}
$$

where $R$ is the resistance of the ESS model; $C$ is the capacitance of the ESS model; and $V_{C}$ is the voltage of the capacitor $C_{\text {ESS. }}$.

For the purpose of the analysis, a simple ESS model is adopted and fixed values of $R$ and $C$ are considered. It is not easy to develop a general model of ESS technologies used for power applications. For instance, the existing models in the literature for chemical battery technologies are generally complicated to set up because of their relative complexity (several parameters to be determined [21]); so a simple first order dynamic circuit is considered appropriate to model the charge and discharge phenomena of the ESS. As mentioned above, the voltage $V_{\text {ESS }}$ represents the SoC of the ESS: when the ESS injects power to the DC-link (discharge phenomena) the voltage decreases, on the contrary in the case of power absorptions (charge phenomena) the voltage increases. The ESS voltage will drop to zero when all the stored energy is supplied; in the same manner it will go to overvoltage values when a big amount of energy is absorbed. These two border operational points will affect the stability and the efficiency of the operation of the ESS converter and the performances of the ESS module; therefore, a lower limit $V_{\text {ESS min }}$ and an upper limit $V_{\mathrm{ESS} \text { max }}$ are introduced in the model.

\section{Study Case}

In this section, the approach proposed is evaluated on a realistic grid model. In particular, the dynamic model of an ESS coupled with a PV system is exploited to study the effect of the primary frequency regulation on the ESS. To this purpose, dynamic RMS simulations are carried out. In the model, the PV system injects a constant amount of power, whereas the ESS injects/absorbs power according to the control strategy of Figure 3. The behaviour of the ESS is analysed by evaluating its response to a real one-day frequency oscillation of the electric system. With this aim, the external grid, modelled as an ideal voltage source, is used to impose a standard one-day frequency oscillation of the ENTSO-E system. The frequency oscillation is represented by a signal of 86400 samples, with a resolution of one sample per second. It is computed starting from data available in [22], where the frequency in the synchronous grid of Continental Europe is measured online. The maximum error of the frequency measurement is defined by the ENTSO-E $(10 \mathrm{mHz})$. The error of the measurement under consideration is below $1 \mathrm{mHz}$, at a frequency of $50 \mathrm{~Hz}$, which corresponds to an accuracy of $0.002 \%$. The one-day frequency oscillation is depicted in Figure 7.

The frequency oscillation vector is computed to obtain the probability density function (PDF), reported in Figure 8. In the same graph the deadband and the maximum deviation $\Delta f_{\max }$ of the frequency control strategy are shown. $97 \%$ of the frequency samples fall inside the $\Delta f_{\max }=50 \mathrm{mHz}$, whereas $61.1 \%$ of the oscillations is inside a deadband equal to $20 \mathrm{mHz}$. Adopting this setting, more than half of the frequency oscillations does not cause any power modulation (samples inside the deadband); on the contrary, only 3\% of the frequency oscillations exceed the $\Delta f_{\max }$ causing a saturation of the control.

The main observation from a study of the data is that the frequency quality in ENTSO-E is maintained very well. Most of the time (61.1\%), the frequency in ENTSO-E stays within the deadband (which is considered as a noncritical window). There are only few frequency excursions outside of $\pm 50 \mathrm{mHz}$ per day. A frequency deviation of $\pm 200 \mathrm{mHz}$ was never reached throughout 2005 [20]. High and low frequency deviations are symmetrical over a long period ( 24 hours). In the short term, however, there are many deviations and they are not necessarily balanced. They can occur at random points in time with random amplitude and random repetition.

4.1. Network Data and Component Sizing. In this section the network data and sizing of components are reported. Typical parameters and standard sizes of the components for this use are adopted. In the following, a list of parameter values adopted for the model is given. 


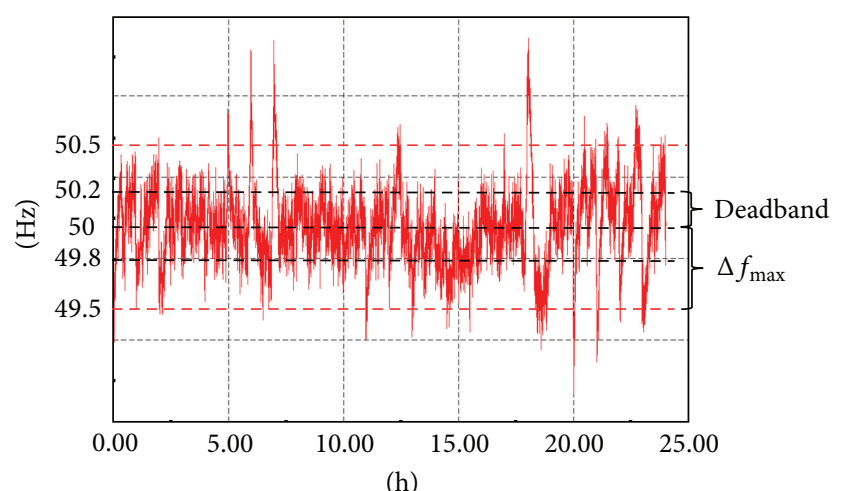

- CM_fctrl: $f$

FIgURE 7: One-day frequency oscillation.

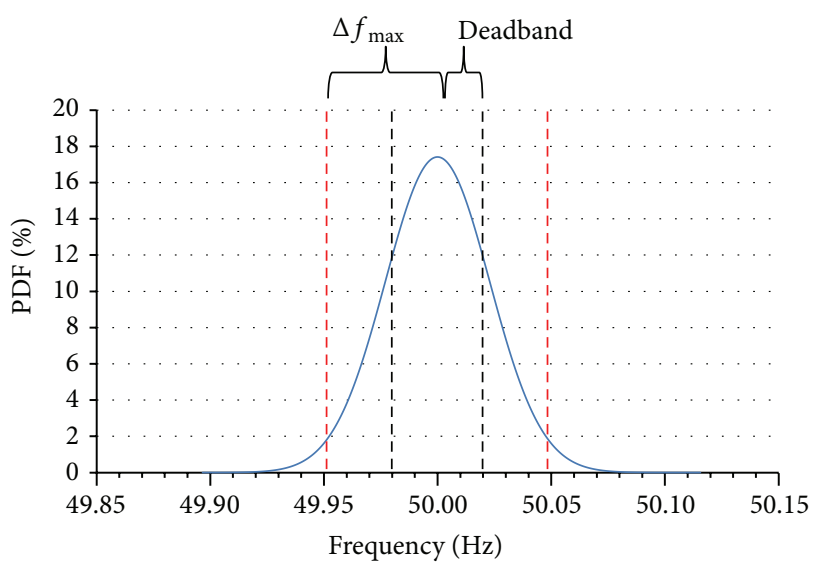

FIGURE 8: PDF of the frequency oscillation vector, deadband and $\Delta f_{\max }$ of the frequency control strategy.

\subsubsection{AC Network}

(i) MV network: $20 \mathrm{kV}$ rated voltage.

(ii) LV network: $400 \mathrm{~V}$ rated voltage.

(iii) INVERTER: $250 \mathrm{kVA}$ rated power and $200 \mathrm{~kW}$ power in the steady state.

(iv) External grid: it operates as voltage and frequency slack, imposing a voltage with constant amplitude of 1.02 p.u. and the frequency oscillations of Figure 7 (it represents a real one-day frequency oscillation, as described in the following).

(v) Data of other elements connected to the AC network are not significant for the purpose of the study.

\subsubsection{Local Control Strategy for Primary Frequency Regulation}

(i) Droop $=2 \%$.

(ii) Deadband $=20 \mathrm{mHz}$.

(iii) $P_{\max }=3 \%$.

The set-point component $\mathrm{DP}_{\text {refESs }}$ related to the SoC restoration function can assume a maximum value $\mathrm{DP}_{\max }$ of
$2.5 \mathrm{~kW}$. The values of droop, deadband, and $\mathrm{DP}_{\max }$ are not imposed by any prescription but they are only adopted as reference settings for the numerical simulation; these settings are changed during the analysis in order to estimate the behaviour of the ESS for different control laws.

\subsubsection{DC-Link}

(i) $700 \mathrm{~V}$ rated voltage.

(ii) $C=30 \mathrm{mF}$.

\subsubsection{PV System}

(i) $1000 \mathrm{~V}$ rated voltage.

(ii) $200 \mathrm{~kW}$ constant power injections equal to the MPPT.

4.1.5. ESS. The ESS capacity is modelled by following the ENTSO-E prescriptions related to the primary frequency control: the storage has to provide the maximum capability $P_{\max }\left(3 \%\right.$ of $\left.P_{n}\right)$ continuously for a period $T$ of 15 minutes.

For the case under analysis, a rated power of $250 \mathrm{~kW}$ implies a maximum energy for the frequency regulation service equal

$$
\begin{aligned}
E & =P \cdot T=0.03 \cdot P_{n} \cdot 15 \cdot 60 \\
& =0.03 \cdot 250 \cdot 10^{3} \cdot 15 \cdot 60=6.75 \mathrm{MJ} .
\end{aligned}
$$

The ESS has to provide the amount of energy of (9) during both a charge and a discharge phenomenon. During the steady-state (constant frequency), the power modulated by the ESS is zero and it should stay at an optimum SoC value in order to guarantee maximum energy both during underfrequency and overfrequency; this optimum value is $50 \%$. It is also assumed an ESS operational range between $20 \%$ and $80 \%$ of its capability, that is, a $20 \%$ margin with respect to a complete charge and discharge. The energy $E$ corresponds to the available band of the SoC between the optimum value and the maximum/minimum limit, and therefore it is $30 \%$ of the theoretical ESS capability. As a consequence of this, the maximum capability of the ESS should be $E_{\max \text { ESS }}=22.5 \mathrm{MJ}$.

Considering a rated voltage of the ESS $V_{\mathrm{ESS} n}=1000 \mathrm{~V}$ (related to a SoC $=100 \%$ ), the minimum capacity $C_{\mathrm{ESS}}$ necessary to satisfy the energetic requirement is $C_{\mathrm{ESS}}=45 \mathrm{~F}$. The minimum suitable capacitance results to be a realistic size for a supercapacitor storage technology; it can be attained by commercially available sizes. Therefore, the series resistance value adopted for the ESS model is set equal to a value of the commercial supercapacitor $(1 \mathrm{~m} \Omega)$ [23].

At this point it is necessary to compute the voltage $V_{\mathrm{ESS}}$ related to each of the aforementioned SoC values; the voltage $V_{\mathrm{ESS} x}$ related to a generic state of charge $\mathrm{SoC}_{x}$ (the latter expressed in \%) is

$$
V_{\mathrm{ESS} x}=\sqrt{\frac{2\left(\mathrm{SoC}_{x} / 100\right) E_{\max \mathrm{ESS}}}{C_{\mathrm{ESS}}}} .
$$

Figure 9 shows the $V_{\text {ESS } x}$ voltage corresponding to a generic $\mathrm{SoC}_{x}$ value. In particular, the voltage corresponding to a SoC $=50 \%$ is $707.1 \mathrm{~V}$ ( 0.7071 p.u. $)$, which corresponds to 


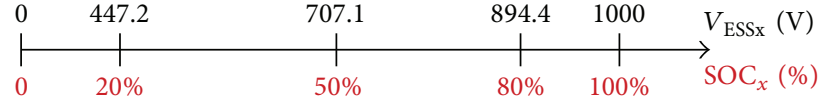

FIgUre 9: Axis of the generic $\operatorname{SoC}_{x}$ values [\%] of the ESS and the corresponding voltage value $V_{\mathrm{ESS} x}[\mathrm{~V}]$.

TABLE 1: PI regulator settings of each converter.

\begin{tabular}{lcc}
\hline Converter & $K$ (p.u.) & $T(\mathrm{~s})$ \\
\hline INVERTER inner loop & 0.2 & 0.005 \\
INVERTER outer loop & 0.05 & 0.015 \\
SOC restoration & 1 & 0.1 \\
MPPT converter & 2 & 0.01 \\
ESS converter & 2 & 0.1 \\
\hline
\end{tabular}

the optimum SoC to guarantee a proper energy margin for the frequency regulation service. A discharge from $50 \%$ to $20 \%$ of the SoC implies a voltage decrease from $707.1 \mathrm{~V}$ to $447.1 \mathrm{~V}$; vice versa, a charge cycle to reach the upper SoC limit of $80 \%$ entails a voltage increase to $894.4 \mathrm{~V}$.

4.1.6. Converter Control Parameters. Proper settings of PI regulators of each static converter are defined in order to satisfy the dynamic requirements of the primary frequency control service. The main dynamic performances according to the ENTSO-E grid code are

(i) to provide $100 \%$ of the required power within 30 seconds,

(ii) to provide $50 \%$ of the required power within 15 seconds.

In Table 1 the control gain $K$ and the time constant $T$ adopted of each PI controller are reported.

4.2. Numerical Analyses. Several dynamic simulations were carried out considering different settings of the frequency control strategy (droop and deadband) and different parameters of the regulator's converters $\left(K_{p}\right.$ and $K_{q}$ gains of the INVERTER outer loop and the $\mathrm{DP}_{\max }$ power available for the SoC restoration function). The modified parameters are reported in Table 2, whereas the rest of the parameters are set to the values reported in Section 3. The aim of the simulation is to verify: (i) the impact of the primary frequency regulation on the ESS and (ii) how this effect varies based on the settings.

On the basis of the settings of Table 2, the probability of the frequency oscillations being within the deadband and $\Delta f_{\max }$ of the frequency control curve is computed (as reported in Table 3).

During the $24 \mathrm{~h}$ simulation the inverter reads the frequency oscillations and modulates the real power on the AC side $P_{\mathrm{AC}}$ according to the frequency control strategy. The real power required for this service is absorbed/injected by the ESS connected to the DC side of the inverter, while the PV system injects a noncontrollable and fixed power.

In order to evaluate the impact of the regulation on the ESS, the power delivered by the ESS is elaborated and the

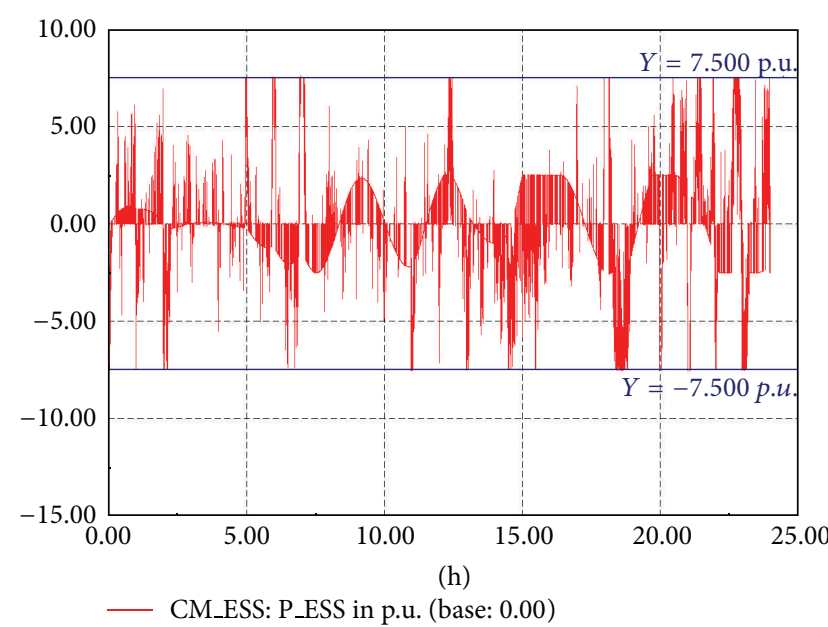

FIGURE 10: One-day power absorbed by the ESS-Set 1.

following energetic indices are computed (they represent the energetic response of the ESS to frequency oscillations).

(i) $E_{\mathrm{ch}}$ : the total charge energy of the ESS.

(ii) $E_{\text {dis }}$ : the total discharge energy of the ESS.

(iii) $E$ : the total energy of the ESS.

(iv) $E_{\text {mch }}$ : the energy margin with respect to the maximum availability $P_{\max }$ during the charge phase.

(v) $E_{\text {mdis }}$ : the energy margin with respect to the maximum availability $P_{\max }$ during the discharge phase.

(vi) $h_{\mathrm{ch}}$ : the equivalent hours at the maximum capability $P_{\max }$ during the charge phase.

(vii) $h_{\text {dis: }}$ the equivalent hours at the maximum capability $P_{\max }$ during the discharge phase.

(viii) $N_{\mathrm{ch}}$ : the equivalent number of complete charge cycles.

(ix) $N_{\text {dis }}$ : the equivalent number of complete discharge cycles.

The real power delivered by the ESS in compliance with the frequency control strategy of Set 1 is shown in Figure 10, while Figure 11 reports the zoom of the power absorbed by the ESS. The two power limits $T 1$ and $T 2$ of the control strategy are highlighted: the former is activated when the maximum power capability available for the frequency control $P_{\max }$ is reached $(7.5 \mathrm{~kW})$ and the latter is activated by the SoC restoration function when the frequency oscillations are within the deadband $(2.5 \mathrm{~kW})$. The voltage of the ESS is shown in Figure 12; it can be noticed that during the one-day power modulation the maximum voltage limit ( 0.849 p.u.) is reached and the saturation flag $K_{\mathrm{ESSSat}}$ of the SoC restoration function (5) becomes zero.

In Table 4, the equivalent charge/discharge cycles of the ESS are reported for each of the settings of Table 2.

The index $N_{\mathrm{ch}}$ is exploited for a comparative analysis of the results obtained by the different settings; this index is directly connected to the energy adopted for the frequency regulation. On the basis of the results of Table 4, it can be 
TABLE 2: Simulation settings.

\begin{tabular}{|c|c|c|c|c|c|c|c|}
\hline & $\begin{array}{c}\text { Droop Df/DP } \\
(\%)\end{array}$ & Deadband $(\mathrm{Hz})$ & $\Delta f_{\max }(\mathrm{Hz})$ & $V_{\text {ESSmin }}$ (p.u.) & $V_{\text {ESSmax }}$ (p.u.) & $\mathrm{DP}_{\max }(\mathrm{MW})$ & $\begin{array}{c}K_{p}-K_{q} \\
\text { P_Q_Ctrl } \\
\text { (p.u./p.u.) }\end{array}$ \\
\hline Set 1 & 2 & 0.02 & 0.05 & 0.8944 & 0.4472 & 0.0025 & 0.05 \\
\hline Set 2 & 2 & 0.01 & 0.04 & 0.8944 & 0.4472 & 0.0025 & 0.05 \\
\hline Set 3 & 2 & 0.04 & 0.07 & 0.8944 & 0.4472 & 0.0025 & 0.05 \\
\hline Set 4 & 1 & 0.02 & 0.035 & 0.8944 & 0.4472 & 0.0025 & 0.05 \\
\hline Set 5 & 4 & 0.02 & 0.08 & 0.8944 & 0.4472 & 0.0025 & 0.05 \\
\hline Set 6 & 2 & 0.02 & 0.05 & 0.8944 & 0.4472 & 0.0025 & 0.8 \\
\hline Set 7 & 2 & 0.02 & 0.05 & 0.8944 & 0.4472 & 0.0025 & 0.015 \\
\hline Set 8 & 2 & 0.02 & 0.05 & 1 & 0 & 0 & 0.05 \\
\hline Set 9 & 2 & 0.02 & 0.05 & 0.8944 & 0.4472 & 0.00025 & 0.05 \\
\hline Set 10 & 2 & 0.02 & 0.05 & 0.8944 & 0.4472 & 0.00125 & 0.05 \\
\hline
\end{tabular}

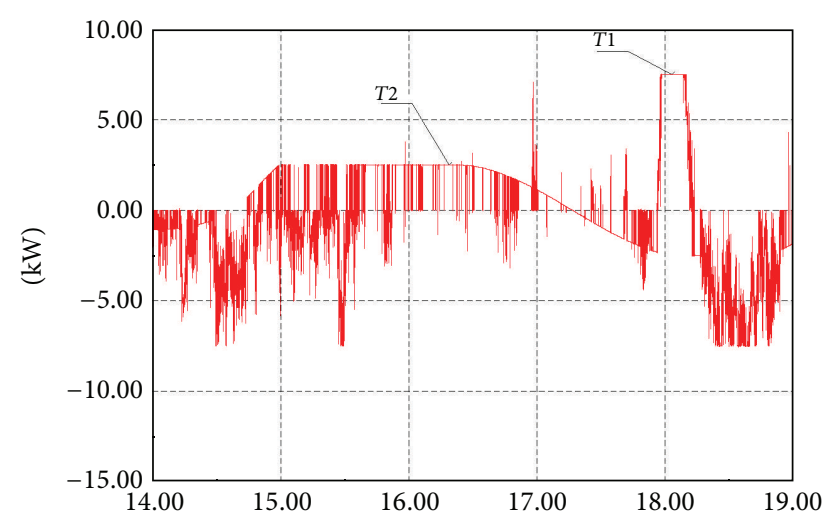

(h)

— CM_ESS: P_ESS in p.u. (base: 0.00)

FIgURE 11: Power absorbed by the ESS in $5 \mathrm{~h}-$ Set 1 .

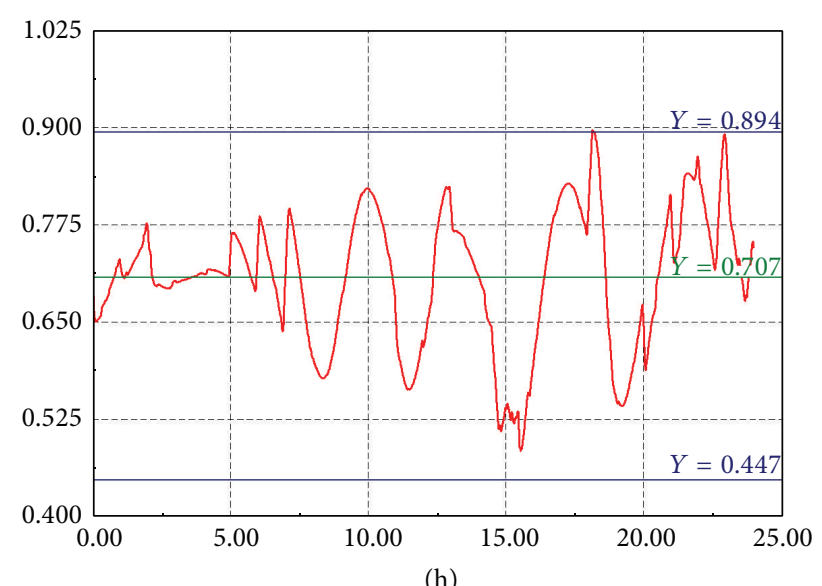

CM_ESS: V_ESS

FIgURE 12: One-day voltage of the ESS-Set 1.
TABLE 3: Probability of the frequency values for different settings.

\begin{tabular}{lcccc}
\hline & $\begin{array}{c}\text { Deadband } \\
(\mathrm{Hz})\end{array}$ & $\begin{array}{c}\% f \text { in } \\
\text { deadband }\end{array}$ & $\Delta f_{\max }(\mathrm{Hz})$ & $\begin{array}{c}\% f \text { in } \\
\Delta f_{\max }\end{array}$ \\
\hline Set 1 & 0.02 & 61.13 & 0.05 & 97 \\
Set 2 & 0.01 & 32.96 & 0.04 & 91.72 \\
Set 3 & 0.04 & 91.72 & 0.07 & 99.78 \\
Set 4 & 0.02 & 61.13 & 0.035 & 87.06 \\
Set 5 & 0.02 & 61.13 & 0.08 & 99.95 \\
Set 6 & 0.02 & 61.13 & 0.05 & 97 \\
Set 7 & 0.02 & 61.13 & 0.05 & 97 \\
Set 8 & 0.02 & 61.13 & 0.05 & 97 \\
Set 9 & 0.02 & 61.13 & 0.05 & 97 \\
Set 10 & 0.02 & 61.13 & 0.05 & 97 \\
\hline
\end{tabular}

stated that the parameters deadband and droop of the curve have a significant impact on the energy modulated by the ESS, in particular:

(i) by increasing the deadband (from Set 1 to Set 3 ) the number of equivalent cycles $N_{\mathrm{ch}}$ strongly decreases (from 5.645 to 2.818), similarly by increasing the deadband value (from Set 1 to Set 2) $N_{\mathrm{ch}}$ increases to 7.484;

(ii) by increasing the droop (from Set 1 to Set 5) the number of equivalent cycles $N_{\mathrm{ch}}$ strongly decreases (from 5.645 to 3.995 ), similarly by increasing this parameters (from Set 1 to Set 4) $N_{\mathrm{ch}}$ increases to 6.530.

The SoC restoration function also affects the energy involved by the ESS: when it is disabled (from Set 1 to Set 8 ) the number of equivalent cycles $N_{\mathrm{ch}}$ decreases (from 5.645 to 3.109) and the ESS is less stressed for charge/discharge cycles; nevertheless, the SoC is not under control and the energy availability for the primary frequency regulation can be compromised. 
TABLE 4: Energetic indices computed from the $P_{\mathrm{ESS}}$ for each simulation setting.

\begin{tabular}{lccccccccc}
\hline Setting & $E_{\text {ch }}(\mathrm{MJ})$ & $E_{\text {dis }}(\mathrm{MJ})$ & $E(\mathrm{MJ})$ & $E_{\text {mch }}(\mathrm{MJ})$ & $E_{\text {mdis }}(\mathrm{MJ})$ & $h_{\mathrm{ch}}$ & $h_{\text {dis }}$ & $N_{\mathrm{ch}}$ & $N_{\text {dis }}$ \\
\hline Set 1 & 76.20 & -74.90 & 1.30 & 571.80 & 573.10 & 2.822 & 2.774 & 5.645 & 5.549 \\
Set 2 & 101.02 & -96.28 & 4.75 & 546.98 & 551.72 & 3.742 & 3.566 & 7.484 & 7.132 \\
Set 3 & 38.05 & -41.25 & -3.20 & 609.95 & 606.75 & 1.409 & 1.528 & 2.818 & 3.056 \\
Set 4 & 90.60 & -88.15 & 2.44 & 557.40 & 559.85 & 3.355 & 3.265 & 6.711 & 6.530 \\
Set 5 & 53.93 & -52.29 & 1.64 & 594.07 & 595.71 & 1.997 & 1.937 & 3.995 & 3.873 \\
Set 6 & 75.71 & -74.21 & 1.50 & 572.29 & 573.79 & 2.804 & 2.748 & 5.609 & 5.497 \\
Set 7 & 75.82 & -74.49 & 1.33 & 572.18 & 573.51 & 2.808 & 2.759 & 5.616 & 5.518 \\
Set 8 & 41.97 & -43.17 & -1.20 & 606.03 & 604.83 & 1.554 & 1.599 & 3.109 & 3.198 \\
Set 9 & 47.72 & -45.07 & 2.66 & 600.28 & 602.93 & 1.767 & 1.669 & 3.535 & 3.338 \\
Set 10 & 63.417 & -60.14 & 3.28 & 584.58 & 587.86 & 2.349 & 2.227 & 4.698 & 4.455 \\
\hline
\end{tabular}

Furthermore, different dynamic responses of the inverter control are tested by acting on the $K_{p}$ and $K_{q}$ gains of the outer loop of the INVERTER. By speeding up the control performances (from Set 1 to Set 6) or slowing down the transient response (from Set 1 to Set 7), the number of equivalent cycles $N_{\mathrm{ch}}$ does not significantly change. It means that the dynamic performances of the control do not affect the ESS energy modulation and lifetime. In Table 5, the time periods in which the $P_{\mathrm{ESS}}$ reaches $100 \%$ and $50 \%$ of maximum capability $P_{\max }$ are reported.

In addition to the energetic indices computed by exploiting the power $P_{\mathrm{ESS}}$, the voltage oscillations at the $R-C$ system $V_{\text {ESS }}$ are analysed in order to evaluate the SoC behaviour during the primary frequency control for each of the settings under study. The minimum value, maximum value, mean value, and standard deviation are reported in Table 6; the monitoring of the voltage $V_{\text {ESS }}$ is very important to supervise the state of the ESS and the availability of energy for the frequency regulation service. The red cells indicate that the maximum or minimum SoC are reached and that the ESS is not able to provide the amount of energy required for the service. The results show that in almost all cases the SoC limits of $20 \%$ and $80 \%$ are reached, and only in the cases in which the ESS is less stressed the SoC does not saturate, that is, with large deadbands (Set 3) and high droops (Set 5).

Table 7 reports for each setting the percentage of samples in which $V_{\text {ESS }}$ exceeds the maximum and minimum thresholds. In the settings with the highest energy involved (Set 2 and Set 4 ), the number of violations is higher than $2 \%$. In Set 8 the SoC restoration function is not activated and the voltage reaches the minimum value for the highest amount of time (10.69\%); this demonstrates the usefulness of an SoC restoration function.

By comparing $E_{\mathrm{ch}}$ and $E_{\mathrm{dis}}$ it can be concluded that, on the basis of the parameters of Set 1, the primary frequency control requires a similar amount of energy both during the charging and the discharging processes. The energy margins $E_{\text {mch }}$ and $E_{\text {mdis }}$ are quite high and the equivalent hours in the one-day simulation $h_{\mathrm{ch}}$ and $h_{\mathrm{dis}}$ are lower than $3 \mathrm{~h}$ (Set 1, Table 4): it means that many partial charge and discharge cycles occur. As a consequence of this, and considering an operative range of $20 \%-80 \%$ of the SoC, 5.645 and 5.549 equivalent charge and discharge cycles per day are completed.
TABle 5: Percentage of $P_{\text {ESS }}$ values at $100 \%$ and $50 \%$ of $P_{\max }$.

\begin{tabular}{lcccc}
\hline Setting & $\begin{array}{c}\%+\_100 \% \\
P_{\max }\end{array}$ & $\begin{array}{c}\%-\_100 \% \\
P_{\max }\end{array}$ & $\begin{array}{c}\%+\_50 \% \\
P_{\max }\end{array}$ & $\begin{array}{c}\%-50 \% \\
P_{\max }\end{array}$ \\
\hline Set 1 & 2.02 & 1.26 & 5.34 & 5.74 \\
Set 2 & 3.14 & 2.82 & 9.56 & 8.89 \\
Set 3 & 0.86 & 0.22 & 1.83 & 0.87 \\
Set 4 & 4.05 & 3.97 & 7.81 & 7.82 \\
Set 5 & 0.46 & 0.03 & 2.29 & 1.56 \\
Set 6 & 2.24 & 1.58 & 5.50 & 5.96 \\
Set 7 & 2.07 & 1.26 & 5.47 & 5.75 \\
Set 8 & 1.82 & 1.00 & 5.22 & 5.66 \\
Set 9 & 2.05 & 1.14 & 5.35 & 5.14 \\
Set 10 & 2.06 & 1.24 & 5.33 & 5.65 \\
\hline
\end{tabular}

TABLE 6: Indices computed from the voltage value $V_{\mathrm{ESS}}$ (equivalent SoC) for each simulation setting.

\begin{tabular}{lcccc}
\hline Setting & Min (p.u.) & Max (p.u.) & Mean (p.u.) & Std (p.u.) \\
\hline Set 1 & 0.4846 & $\mathbf{0 . 8 9 6 3}$ & 0.7073 & 0.0866 \\
Set 2 & $\mathbf{0 . 4 4 3 4}$ & $\mathbf{0 . 9 0 4 4}$ & 0.6964 & 0.1052 \\
Set 3 & 0.5964 & 0.7895 & 0.7068 & 0.0485 \\
Set 4 & $\mathbf{0 . 4 3 0 9}$ & $\mathbf{0 . 8 9 8 6}$ & 0.7010 & 0.1073 \\
Set 5 & 0.5293 & 0.8831 & 0.7140 & 0.0697 \\
Set 6 & 0.4819 & $\mathbf{0 . 8 9 6 3}$ & 0.7075 & 0.0863 \\
Set 7 & 0.4844 & $\mathbf{0 . 8 9 6 3}$ & 0.7074 & 0.0865 \\
Set 8 & $\mathbf{0 . 2 4 2 5}$ & $\mathbf{0 . 8 9 4 6}$ & 0.6755 & 0.1633 \\
Set 9 & $\mathbf{0 . 4 4 3 4}$ & $\mathbf{0 . 8 9 6 2}$ & 0.6810 & 0.1324 \\
Set 10 & $\mathbf{0 . 4 4 3 9}$ & $\mathbf{0 . 8 9 6 2}$ & 0.6971 & 0.0832 \\
\hline
\end{tabular}

The available storage solutions can manage 5000 charge and discharge cycles, which means that if they are employed for this application they last 2.4 years. The setting values also play an important role in the amount of energy involved by the ESS: the system lifetime can be even lower if the ESS is more involved in the service; on the contrary, it could be higher but paying with lower regulation performances. The ESS lifetime comparison for each simulation setting is reported in Table 8 .

This application may have an important impact on the storage life duration. Therefore, the simulation outcomes $N_{\mathrm{ch}}$ 
TABLE 7: Percentage of $V_{\text {ESS }}$ values $V_{\text {ESS max }}$ and $V_{\text {ESS min }}$.

\begin{tabular}{lcc}
\hline Setting & $\% u+\_100 \% u_{\max }$ & $\% u-\_100 \% u_{\min }$ \\
\hline Set 1 & 0.19 & 0 \\
Set 2 & 2.76 & 2.40 \\
Set 3 & 0 & 0 \\
Set 4 & 2.21 & 2.15 \\
Set 5 & 0 & 0 \\
Set 6 & 0.26 & 0 \\
Set 7 & 0.18 & 0 \\
Set 8 & 1.38 & 10.69 \\
Set 9 & 0.35 & 1.80 \\
Set 10 & 0.42 & 0.19 \\
\hline
\end{tabular}

TABLE 8: ESS lifetime for each simulation setting.

\begin{tabular}{lc}
\hline Setting & ESS lifetime (years) \\
\hline Set 1 & 2.4 \\
Set 2 & 1.9 \\
Set 3 & 4.7 \\
Set 4 & 2.1 \\
Set 5 & 3.5 \\
Set 6 & 2.5 \\
Set 7 & 2.5 \\
Set 8 & 4.3 \\
Set 9 & 4 \\
Set 10 & 3 \\
\hline
\end{tabular}

and $N_{\text {dis }}$ are useful for an economic and technical evaluation of the ESS sizing. Anyway, a suitable trade-off between the frequency regulation service and the ESS costs is needed.

Finally, a stability issue is worth noting for electric grids characterised by a decentralised control of a large amount of DG. Reference [24] demonstrates that DGs may start interacting with each other and/or with conventional power plants, leading to small-signal instability problems, but these phenomena are limited to electrically close generators. To ensure stability of future distribution systems, new centralised and decentralised control schemes will be required. One of the possible solutions for the short-term scenario is to introduce a time-constant decoupling between conventional power plants and DG; in particular, as in the work reported in this paper, exploiting the fast dynamic of the ESS in order to perform frequency control faster. As detailed in Table 4, such a solution does not significantly affect the ESS energy modulation and lifetime. Moreover, in order to avoid DG oscillations, DSOs (distribution system operators) will have to technically check the local distribution grid in order to avoid the connection of ESSs electrically close to each other.

\section{Conclusion}

The provision of an adequate power reserve through DG power plants and ESSs is important for a secure network: it allows TSOs to keep the power system load and generation always balanced, avoiding/limiting frequency deviations from the rated value.

This work explores the effect on the ESS related to the frequency control to be extended to renewable DG units so that they can provide the frequency regulation service like a conventional generator.

The study also shows that the control settings are other important parameters that contribute to the ESS performances and lifetime duration. The results of the numerical simulations show that charge/discharge frequency, expressed as number of daily equivalent charge/discharge cycles, can be relatively high in this application and, hence, the service means the apparatus involved has a relevant effect in terms of lifetime, maintenance, and operation costs.

The control scheme considered introduces the SoC restoration concept to restore the optimal energy state of the ESS. The SoC restoration function makes a compromise because it deteriorates the ESS lifetime in order to guarantee a full utilisation of the ESS capacity for the frequency regulation service.

Finally, note that this paper focuses on the impact of the frequency regulation service, which could become a mandatory task for all power plants to the ESS and on how its control should be designed. Hence, this paper could be useful to provide the engineering data needed for the economic assessment of deploying such an ESS in practice for network service applications.

\section{Conflict of Interests}

The authors declare that they have no conflict of interests regarding the publication of this paper.

\section{References}

[1] S. Teleke, M. E. Baran, A. Q. Huang, S. Bhattacharya, and L. Anderson, "Control strategies for battery energy storage for wind farm dispatching," IEEE Transactions on Energy Conversion, vol. 24, no. 3, pp. 725-732, 2009.

[2] G. Callegari, P. Capurso, F. Lanzi, M. Merlo, and R. Zaottini, "Wind power generation impact on the frequency regulation: study on a national scale power system," in Proceedings of the 14th International Conference on Harmonics and Quality of Power (ICHQP '10), pp. 1-6, Bergamo, Italy, September 2010.

[3] CIGRE, Ancillary Services: An Overview of International Practices, CIGRE Working Group C5. 06, 2010.

[4] "Survey on Ancillary Services Procurement \& Balancing market design," 2012, https://www.entsoe.eu/fileadmin/user_upload/_ library/resources/BAL/121022_Survey_on_AS_Procurement_ and_EBM_design.pdf

[5] R. Raineri, S. Ríos, and D. Schiele, “Technical and economic aspects of ancillary services markets in the electric power industry: an international comparison," Energy Policy, vol. 34, no. 13, pp. 1540-1555, 2006.

[6] P. Cappers, A. Mills, C. Goldman, R. Wiser, and J. H. Eto, "An assessment of the role mass market demand response could play in contributing to the management of variable generation integration issues," Energy Policy, vol. 48, pp. 420-429, 2012. 
[7] P. Cappers, J. MacDonald, C. Goldman, and O. Ma, "An assessment of market and policy barriers for demand response providing ancillary services in U.S. electricity markets," Energy Policy, vol. 62, pp. 1031-1039, 2013.

[8] F. Del Pizzo and CEO Terna Storage, Build-Up of Italian Power Market and Interconnections to Neighboring Systems, Dubai, United Arab Emirates, 2013.

[9] GSE Gestore Servizi Energetici, Statistical report 2012, Renewable power plants-Electrical sector, in Italian.

[10] J. Eto, J. Undrill, P. Mackin et al., Use of Frequency Response Metrics to Assess the Planning and Operating Requirements for Reliable Integration of Variable Renewable Generation, Lawrence Berkeley National Laboratory, Berkeley, Calif, USA, 2010.

[11] P. F. Ribeiro, B. K. Johnson, M. L. Crow, A. Arsoy, and Y. Liu, "Energy Storage systems for Advances Power Applications," Proceedings of the IEEE, vol. 89, no. 12, pp. 1744-1756, 2001.

[12] Bonneville Power Administration, "Renewable Energy Technology Roadmap, Technology Innovation Office," 2006, http:// www.bpa.gov/corporate/business/innovation/docs/2006/RM06_Renewables-Final.pdf.

[13] K. Yoshimoto, N. Toshiya, G. Koshimizu, and Y. Uchida, "New control method for regulating State-of-Charge of a battery in hybrid wind power/battery energy storage system," in Proceedings of the IEEE PES Power Systems Conference and Exposition (PSCE '06), pp. 1244-1251, Atlanta, Ga, USA, November 2006.

[14] I. Serban and C. Marinescu, "An enhanced three-phase battery energy storage system for frequency control in microgrids," in Proceedings of the 13th International Conference on Optimization of Electrical and Electronic Equipment (OPTIM '12), pp. 912-918, Brasov, Romania, May 2012.

[15] Italian Electrical Committee (CEI), Technical Standard CEI 016, Reference Technical Rules for the Connection of Active and Passive Consumers to the HV and MV Electrical Networks of Distribution Company, CEI, Milan, Italy, 2012.

[16] R. Doherty, A. Mullane, G. Nolan, D. J. Burke, A. Bryson, and M. O'Malley, "An assessment of the impact of wind generation on system frequency control," IEEE Transactions on Power Systems, vol. 25, no. 1, pp. 452-460, 2010.

[17] ENTSO-E, "Network Code for Requirements for Grid Connection applicable to all Generators," 2012, https://www.entsoe.eu.

[18] A. H. M. A. Rahim and M. A. Alam, "Fast low voltage ridethrough of wind generation systems using supercapacitor based energy storage systems," in Proceedings of the 4th International Conference on Modeling, Simulation and Applied Optimization (ICMSAO '11), pp. 1-6, Kuala Lumpur, Malaysia, April 2011.

[19] P. Srithorn, M. Sumner, L. Yao, and R. Parashar, "The control of a statcom with supercapacitor energy storage for improved power quality," in Proceedings of the CIRED Seminar 2008: SmartGrids for Distribution, Frankfurt, Germany, June 2008.

[20] A. Oudalov, D. Chartouni, and C. Ohler, "Optimizing a battery energy storage system for primary frequency control," IEEE Transactions on Power Systems, vol. 22, no. 3, pp. 1259-1266, 2007.

[21] M. B. Camara, H. Gualous, F. Gustin, and A. Berthon, "Design and new control of DC/DC converters to share energy between supercapacitors and batteries in hybrid vehicles," IEEE Transactions on Vehicular Technology, vol. 57, no. 5, pp. 2721-2735, 2008.

[22] "Netzfrequenzmessung. Beispieltag der Netzfrequenzmessung mit Minuten- und Sekundenwerten," 2011, https://www.netzfrequenzmessung.de
[23] V. Musolino, L. Piegari, and E. Tironi, "New full-frequencyrange supercapacitor model with easy identification procedure," IEEE Transactions on Industrial Electronics, vol. 60, no. 1, pp. 112-120, 2013.

[24] M. Honarvar Nazari, M. Ilic, and J. Peças Lopes, "Small-signal stability and decentralized control design for electric energy systems with a large penetration of distributed generators," Control Engineering Practice, vol. 20, no. 9, pp. 823-831, 2012. 


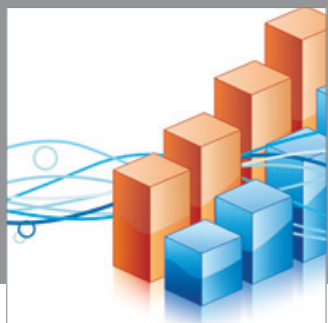

Advances in

Operations Research

mansans

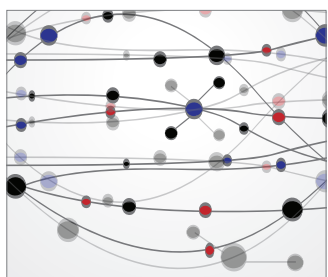

The Scientific World Journal
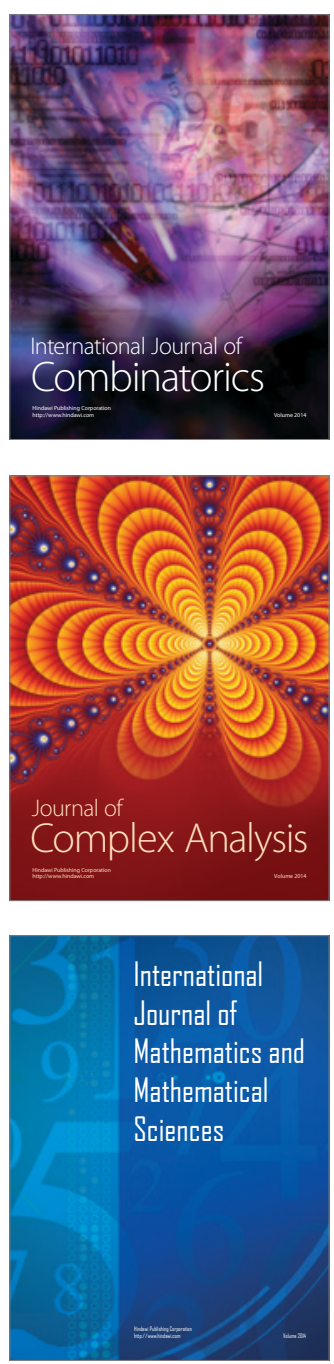
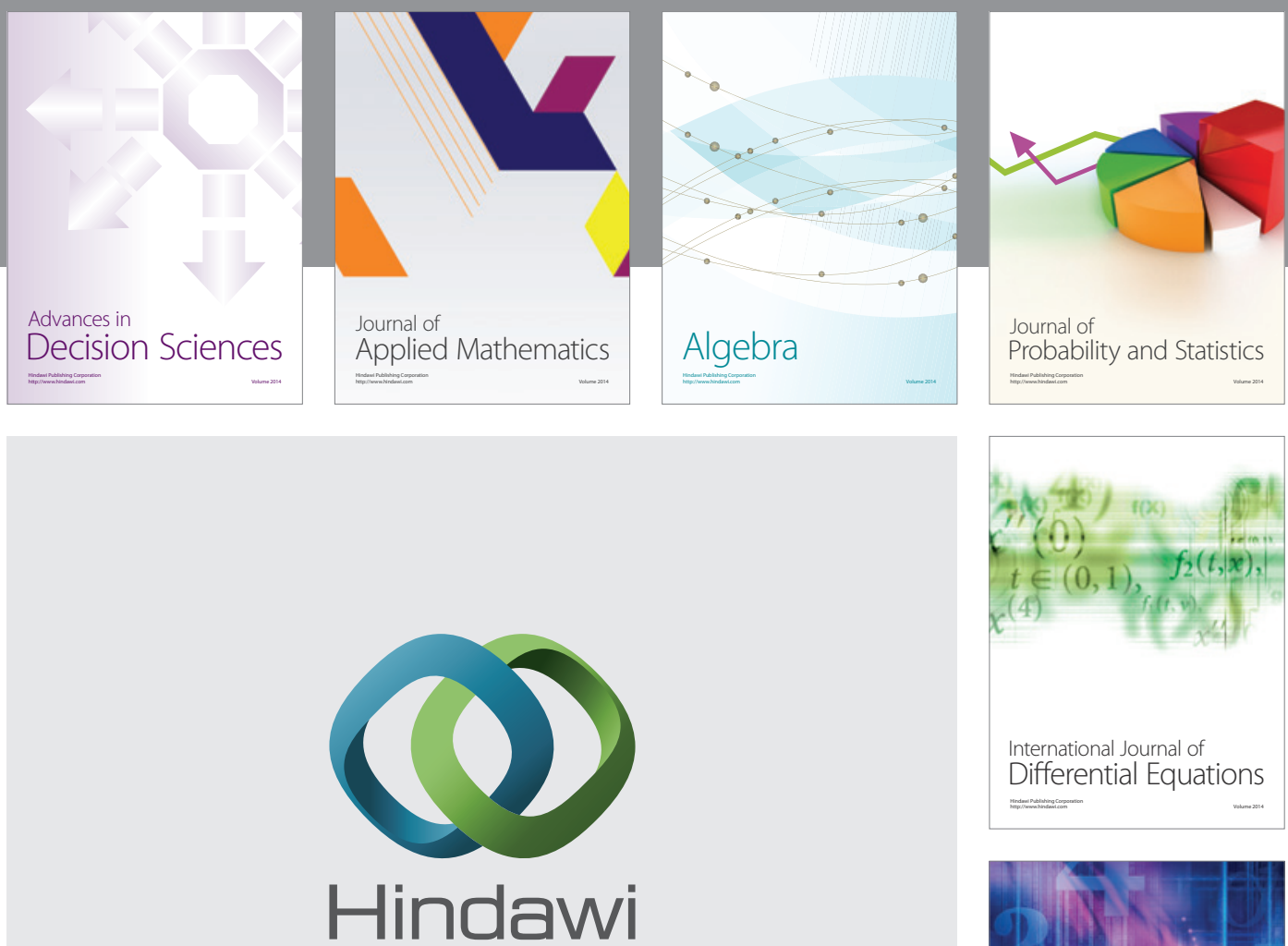

Submit your manuscripts at http://www.hindawi.com
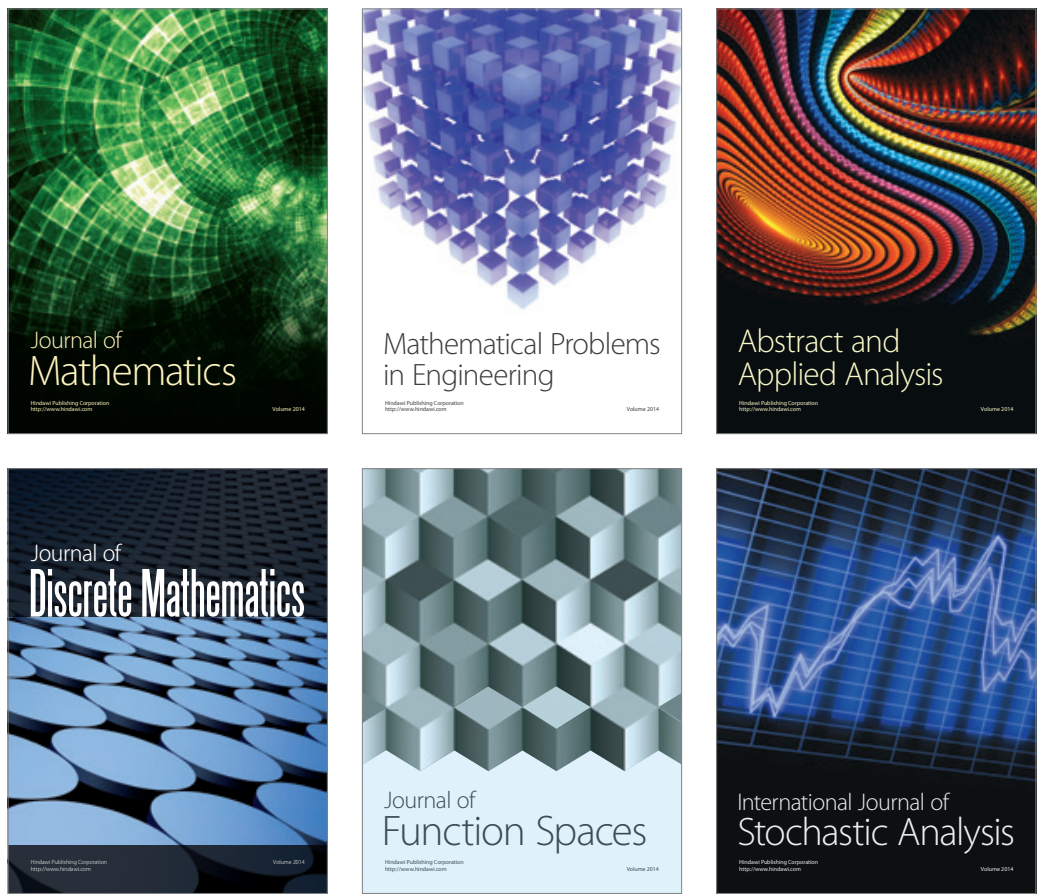

Journal of

Function Spaces

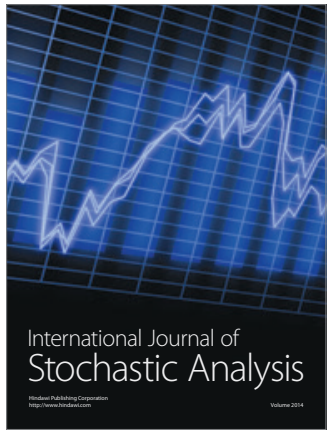

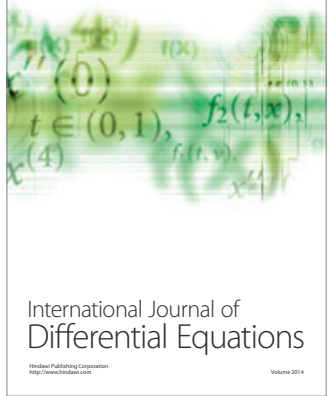
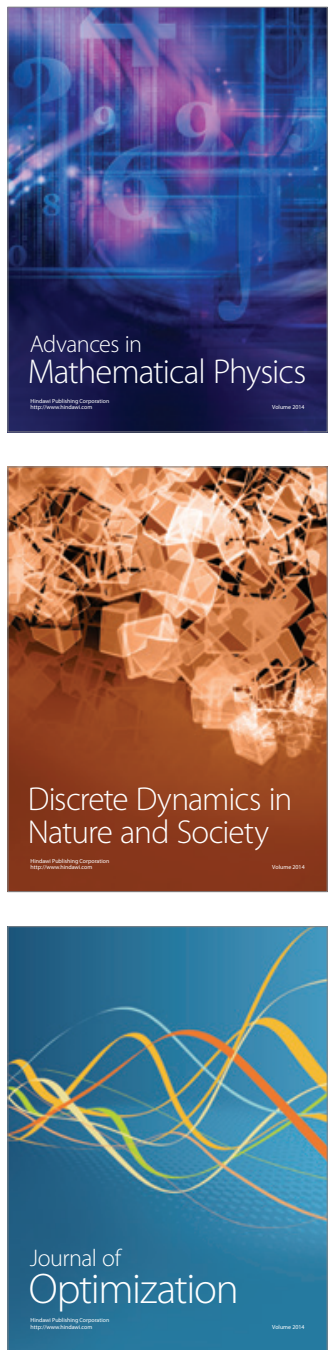